\title{
ARTICLE
}

\section{Development of the Quasi-monoenergetic Neutron Calibration Fields of Several Tens of MeV at TIARA}

\author{
Yoshiaki SHIKAZE ${ }^{1, *}$, Yoshihiko TANIMURA ${ }^{1}$, Jun SAEGUSA ${ }^{1}$, Masahiro TSUTSUMI ${ }^{1}$, \\ Yoshiaki UCHITA ${ }^{1}$ and Michio YOSHIZAWA ${ }^{1}$ \\ ${ }^{1}$ Japan Atomic Energy Agency, Tokai, Naka, Ibaraki 319-1195, Japan
}

\begin{abstract}
Development of three calibration fields for 45, 60 and $75 \mathrm{MeV}$ neutrons has been in progress at Takasaki Ion Accelerators for Advanced Radiation Application (TIARA) of JAEA-Takasaki. Achievement of the neutron calibration fields requires establishment of both measurement of neutron fluence and its monitoring technique. In order to measure the neutron fluence of main energy peak especially at a reference point on low fluence condition, a proton recoil counter telescope with high efficiency has been developed. The design of relatively large telescope components brought a high detection efficiency at the reference point with wide irradiation area at a long distance. Effective and precise measurement of the neutron fluence could be performed with uncertainties less than $6.5 \%$ (standard deviation). For detecting neutrons without scattering near the target, a transmission type neutron fluence monitor has been newly developed to measure the neutron fluence directly at the collimator exit. Its performance such as counting rates was tested for various beam intensities.
\end{abstract}

KEYWORDS: neutron calibration field, TIARA, proton recoil counter telescope, fluence monitor

\section{Introduction*}

High energy proton accelerator facilities such as J-PARC have been developed to pursue frontier research in particle physics, nuclear science and technology. In order to implement the quality of radiation protection in such facilities, neutron calibration fields in a wide energy range are required to evaluate the energy response of neutron monitors and dosimeters.

For the neutron fields above $20 \mathrm{MeV}$, the standard calibration fields have not been fully established in Japan. For the purpose of the development of such calibration fields, the quasi-monoenergetic neutron irradiation fields of several tens of $\mathrm{MeV}$ are available at Takasaki Ion Accelerators for Advanced Radiation Application (TIARA) of Takasaki Advanced Radiation Research Institute (TARRI), Japan Atomic Energy Agency (JAEA). ${ }^{1}$ Therefore, investigation of the characteristics of the neutron fields at TIARA ${ }^{2,3)}$ has been furthered in order to contribute to the establishment of the standard fields by the National Metrology Institute of Japan, National Institute of Advanced Industrial Science and Technology (AIST), in the future. Three neutron fields with 45, 60 and $75 \mathrm{MeV}$ peaks are planned to be established, considering the international intercomparison of the neutron fields at TIARA with those at other facilities in a similar energy range.

Up to now, beam profile, main peak energy of quasi-monoenergetic neutron, and neutron spectra inside and outside the irradiation field were measured and reported.,3) Among remaining issues on neutron fluence for the main energy peak and on corrections for low energy neutrons below the main energy peak region and gamma rays, measurement of neutron fluence and establishment of

*Corresponding Author, E-mail:shikaze@jaea.go.jp

(C) Atomic Energy Society of Japan fluence monitoring technique are important for the development of the calibration fields. This paper reports the results of absolute measurement of neutron fluence by using a proton recoil counter telescope (PRT) with high detection efficiency and the development of a transmission type neutron fluence monitor to monitor neutron beam directly.

\section{Quasi-monoenergetic Neutron Fields at TIARA}

The quasi-monoenergetic neutron fields by using ${ }^{7} \mathrm{Li}(\mathrm{p}, \mathrm{n}){ }^{7} \mathrm{Be}$ reaction for $40-90 \mathrm{MeV}$ neutrons have been established at TIARA as the irradiation field for experimental use. ${ }^{1)}$ The neutron spectra have the main energy peak in high energy region and continuous distribution below the peak region. Figure 1 shows the layout of the quasi-monoenergetic neutron source facility at TIARA. A proton beam from an AVF cyclotron is transported to a ${ }^{7} \mathrm{Li}$ target with thickness corresponding to $2 \mathrm{MeV}$ energy loss. Protons passing through the target are bent by a clearing magnet into a Faraday cup shielded by an iron beam dump. Neutrons are guided to the experimental room through about $3 \mathrm{~m}$ thick collimator (inner-diameter of $11 \mathrm{~cm}$ ) consisting of an iron rotary-shutter

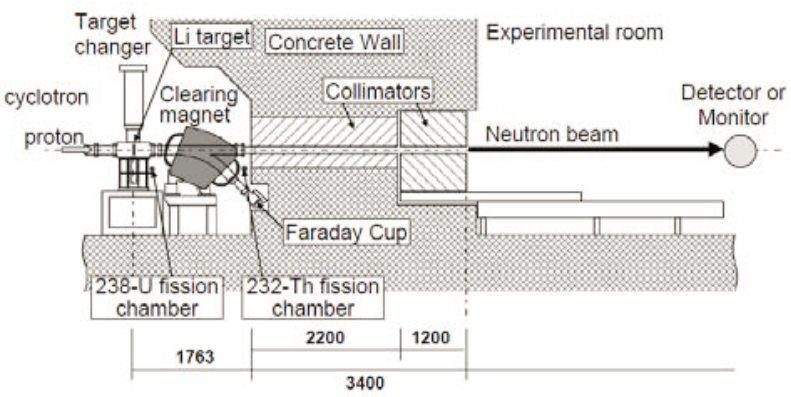

Fig. 1 Schematic view of the quasi-monoenergetic neutron source facility at TIARA. 
and additional polyethylene and iron collimators. Neutron beam in the experimental room, therefore, has low background of neutrons scattered by the materials around the target and ahead of the collimators. The dimensions of the experimental room are $11 \mathrm{~m}(\mathrm{~W}) \times 19 \mathrm{~m}(\mathrm{D}) \times 6 \mathrm{~m}(\mathrm{H})$. The distance from the ${ }^{7} \mathrm{Li}$ target ranges from 5 to $18 \mathrm{~m}$ at on-beam position in the room. Currently, the Faraday cup in the beam dump, ${ }^{238} \mathrm{U}$ and ${ }^{232} \mathrm{Th}$ fission chambers placed around the target are available neutron monitors (Fig. 1).

\section{Development of Neutron Fluence Measurement Technique}

\section{Absolute Measurement of Neutron Fluence}

(1) Development of a Proton Recoil Counter Telescope

Neutron fluence in the main energy peak region is one of the most important parameters of the neutron calibration field with quasi-monoenergetic spectrum. A point $8 \mathrm{~m}$ away from the collimator exit on low fluence condition was determined to be the reference point that would supply a sufficient irradiation area for calibrating the large high energy neutron monitors and dosimeters. The PRT detectors and converters made by the Baba group ${ }^{1)}$ were too small to measure the neutron fluence at the reference point with sufficient count statistics during the limited beam time. Therefore, a PRT with higher efficiency for use at the reference point was newly developed to make the absolute measurement of the peak neutron fluence.

The PRT consists of annular converters (polyethylene and graphite ones), a silicon semi-conductor detector (PIPS, $62 \mathrm{~mm}$ in dia., $300 \mu \mathrm{m}$ thick) as $\Delta \mathrm{E}$ detector, an organic liquid scintillation detector (BC501A, $7.62 \mathrm{~cm}$ (L) x $7.62 \mathrm{~cm}$ in dia.) as $\mathrm{E}$ detector and a brass cylinder for the shield of the sensitive volumes of the detectors from neutron beam. In front of the detectors, a brass aperture with $50 \mathrm{~mm}$ in dia. is attached.

The choice of the organic liquid scintillator is based on both the merits of being less sensitive to gamma rays than $\mathrm{NaI}(\mathrm{Tl})$ detectors and the fast timing signals used for the TOF measurement. As for the first merit, a larger volume E detector was required for high efficiency. If the $\mathrm{NaI}(\mathrm{Tl})$ as the $\mathrm{E}$ detector becomes large, signal-to-noise ratio becomes worse because of higher sensitivity to gamma rays. The problem prevented us from making a large $\mathrm{E}$ detector with $\mathrm{NaI}(\mathrm{Tl})$, but the organic liquid scintillator with less sensitivity to gamma rays and capability of neutron-gamma separation could become a large $\mathrm{E}$ detector. As for the second merit, the TOF data can be supplementarily used to increase the reliability of recoil proton measurements. This is based on separation between recoil protons and background protons in the proton energy v.s. TOF distribution after selecting proton event in the $\Delta \mathrm{E}$ v.s. $\mathrm{E}$ distribution. The choice is unique among $\mathrm{E}$ detectors of the same type of PRTs such as $\mathrm{NaI}(\mathrm{Tl}), \mathrm{CsI}(\mathrm{Tl})$ and Si detectors, which cannot simultaneously measure TOF data at very good timing resolution.

In the measurement, the PRT components were set in the irradiation field on the beam axis as shown in Fig. 2. Distance between the converter and the aperture was selected so that recoil protons produced in the polyethylene converter and passing through the aperture could enter and fully stop in the

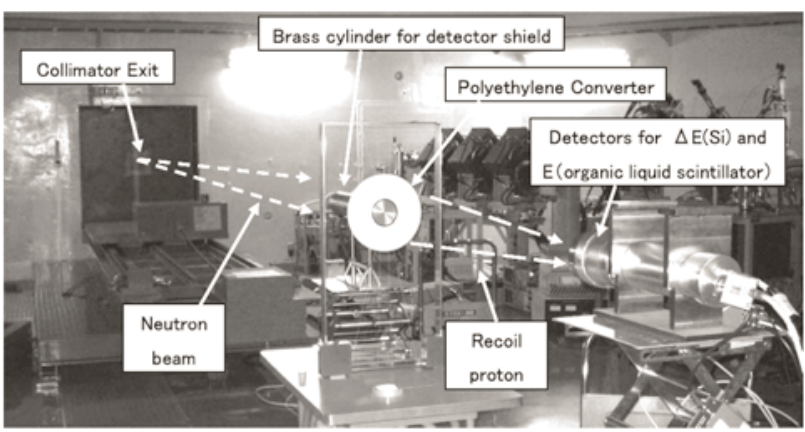

Fig. 2 Layout of each component of the PRT for the absolute measurement in the neutron irradiation room at TIARA.

scintillator. Since the irradiation field at the reference point had about $30 \mathrm{~cm}$ in diameter, the annular converters with inner and outer diameters of 8.8 and $25.0 \mathrm{~cm}$, respectively, were prepared to make the efficiency high.

\section{(2) Analysis of Recoil Proton Spectrum}

The PRT measures the recoil protons from the n-p elastic scattering in a polyethylene converter. Proton events are clearly identified in the $\Delta \mathrm{E}$ v.s. E distribution among gamma-ray and other charged particle events. By setting boundary of the region for protons, proton events are selected. Then, proton spectrum is obtained as a projection of selected proton events to the E-axis. The influence caused by the nuclear interactions of carbon in the polyethylene converter and by those of air in the irradiation field is corrected by using measurement with the graphite converter. For the graphite converter data, proton spectrum can be obtained in the same way as the polyethylene converter data. Before the subtraction of background protons generated from carbon and air, both proton spectra are normalized by the beam current during the measurements. After the normalization and the background subtraction, recoil proton spectrum can be obtained.

Neutron detection efficiency of the PRT was estimated by using the Monte Carlo code MCNPX with the LA150 data set. From the number of recoil protons $(\mathrm{N})$, beam current $(\mathrm{Q})$, an area of the converter (S), distance from the target (D) and detection efficiency calculated by the MCNPX code $\left(\varepsilon_{\text {eff }}\left(E_{n}\right)\right)$, neutron spectral fluence $\left(\Phi\left(\mathrm{E}_{\mathrm{n}}\right)\right)$ can be obtained in unit of neutrons per unit steradian per unit beam current $([\mathrm{n} / \mathrm{sr} / \mu \mathrm{C}])$ by the following equation:

$$
\Phi\left(E_{n}\right)=N / \varepsilon_{\text {eff }}\left(E_{n}\right) / S \times D^{2} / Q
$$

\section{(3) Measurement of Neutron Fluence}

The developed PRT was used to measure fluences in the neutron fields at TIARA, where neutrons were produced in the nuclear reaction of ${ }^{7} \mathrm{Li}$ targets with 50,65 and $80 \mathrm{MeV}$ protons (The proton energies correspond to the neutrons with the main peak energy of 45, 60 and $75 \mathrm{MeV}$, respectively.). For the measurement of the neutron fluence at a reference point (12.905 $\mathrm{m}$ from the target), the converter were set at $8.0 \mathrm{~m}$ from the collimator exit. Measurements with polyethylene converter and those with graphite converter were performed with the proton beam current of $0.5-1.2 \mu \mathrm{A}$ and the irradiation period of 1-1.5 
hours. During the irradiation period, about 5000-11000 recoil proton events were obtained in the peak region of the recoil proton energy spectra after background subtraction.

(4) Results

By using Eq.(1), neutron fluences in the peak region were obtained as $2.89 \times 10^{9}, 3.91 \times 10^{9}$ and $4.93 \times 10^{9}[\mathrm{n} / \mathrm{sr} / \mu \mathrm{C}]$ for 45, 60 and $75 \mathrm{MeV}$, respectively. These neutron fluences were the averaged values over 2 or 3 measurements under different experimental conditions with the same peak energy but with the slightly different target thickness. The neutron fluences here were corrected by each thickness of the ${ }^{7} \mathrm{Li}$ target so as to correspond to thicknesses of 3.80, 5.00 and $6.00 \mathrm{~mm}$ for 45,60 and $75 \mathrm{MeV}$, respectively. It should be also noted that the neutron fluences were measured under the condition that the transmission type neutron fluence monitor described in the next section was set on the collimator exit.

The neutron fluences are summarized in Table $\mathbf{1}$ with uncertainties. As uncertainties of the neutron fluence, the following components were considered; (1) counting statistics of recoil protons, (2) n-p scattering cross section, (3) statistics of detection efficiency calculation, (4) geometry, (5) statistics of beam monitor and (6) normalization of beam current. The standard uncertainties $(\mathrm{k}=1)$ were estimated, and by combining each uncertainty the total ones were obtained as noted in the parentheses of Table 1, where $\mathrm{k}$ is a coverage factor (uncertainty for $\mathrm{k}=1$ is equal to the standard deviation, with confidence level for the uncertainty being 68.27\%).

From these measurements, it was confirmed that detection efficiency of the PRT was enough to make the absolute measurements of the peak neutron fluence at the reference point (12.905 $\mathrm{m}$ from the target) of the neutron beam line at TIARA.

Table 1: Summary of the neutron fluence of the neutron beam line at TIARA

\begin{tabular}{|c|c|c|c|}
\hline $\begin{array}{c}\text { Energy of } \\
\text { main peak } \\
{[\mathrm{MeV}]}\end{array}$ & $\begin{array}{c}{ }^{7} \mathrm{Li} \text { target } \\
\text { thickness } \\
{[\mathrm{mm}]}\end{array}$ & $\begin{array}{c}\text { Maximum } \\
\text { beam current } \\
{[\mu \mathrm{A}]}\end{array}$ & $\begin{array}{c}\text { Fluence } \\
\text { (uncertainty[k=1]) } \\
{[\mathrm{n} / \mathrm{sr} / \mu \mathrm{C}]}\end{array}$ \\
\hline 45 & 3.80 & 3.4 & $2.89 \times 10^{9}( \pm 6.2 \%)$ \\
\hline 60 & 5.00 & 2.0 & $3.91 \times 10^{9}( \pm 6.2 \%)$ \\
\hline 75 & 6.00 & 1.2 & $4.93 \times 10^{9}( \pm 6.5 \%)$ \\
\hline
\end{tabular}

\section{Development of the Neutron Fluence Monitor}

(1) Motivation of the Development

In practical calibration at the neutron field by using an accelerator, neutron fluence must be continuously monitored with sufficient precision during the irradiation period. At TIARA, neutrons induced by the nuclear reactions are transported into the irradiation room through the collimator. However, the existing neutron fluence monitor with the fission chamber is located around the neutron beam line (off-line) and ahead of the collimator (Fig. 1). The existing monitor cannot monitor directly neutrons in the irradiation room. The existing monitor is influenced by unwanted neutrons which are differently produced at every experiment from the beam transport line and from the materials around the monitor. Therefore, a prototype of a transmission type neutron fluence monitor has been developed ${ }^{4}$ to monitor directly the neutrons transported into the irradiation room (on-line). The prototype adopted a thin plastic scintillator and acrylic light guides on its both sides coated by reflection material. They were installed at the center of an aluminum case. The previous performance test indicated that low collection efficiency of scintillation lights led to lower sensitivity than the existing monitor (fission chamber). Therefore, in order to increase sensitivity, a transmission type neutron fluence monitor with different structure has been newly developed by improving light collection efficiency.

In most of the neutron fields in the world, proton beam current is measured with the Faraday Cup. In some of the neutron fields, the transmission type fission chamber is used as a beam intensity monitor. As a transmission type monitor, the plastic scintillator with a thickness of $0.5 \mathrm{~mm}$ is used only for small irradiation field (for example, the irradiation field with a diameter of $30 \mathrm{~mm}$ ). The small size of the scintillator makes attenuation of scintillation lights very small and light collection easy. In the neutron field at TIARA, the irradiation field size at the collimator exit is $109 \mathrm{~mm}$ in diameter. Improvement of the light collection is required to monitor all the area of the relatively large irradiation field since attenuation of the thin scintillator is more rapid than the scintillators of small size used in other fields.

(2) Development of the New Fluence Monitor

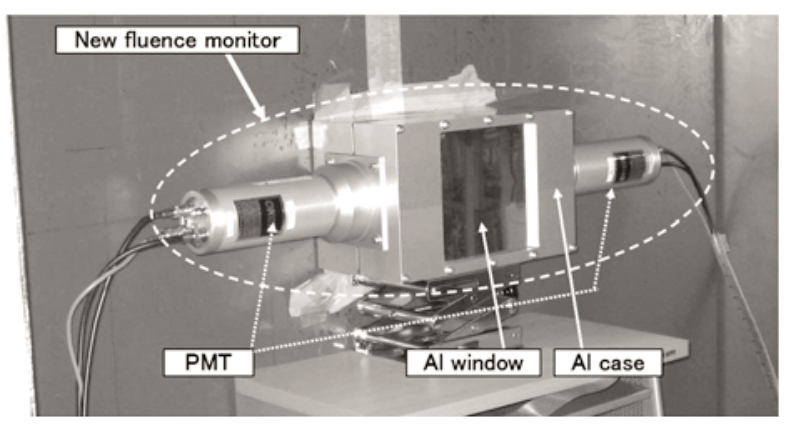

Fig. 3 New neutron fluence monitor consists of a plastic scintillator without coating, an aluminum case with aluminum entrance windows and two PMTs.

The new monitor consists of a thin plastic scintillator $\left(\mathrm{BC} 400,150 \times 150 \times 0.5 \mathrm{~mm}^{3}\right)$, an aluminum case with thin aluminum entrance and exit windows (145 x $\left.145 \times 0.5 \mathrm{~mm}^{3}\right)$ and two photomultiplier tubes (PMTs) (Fig. 3). The new monitor was designed to detect mainly the recoil protons generated in the scintillator by the neutron beam. For choice of the scintillator thickness, larger scintillator thicknesses yield higher detection efficiencies, but they make transmission losses of neutrons worse, increasing dead time and scattered neutrons of low energies. The scintillator thickness of $0.5 \mathrm{~mm}$ was selected so that the sensitivity was the same level as the existing monitors and the transmission loss within $1 \%$ by the calculations using the MCNPX code. The PMTs on both sides of the aluminum case detect the scintillation light yielded in the scintillator. The scintillator installed in the aluminum case is fixed on the inner surface of the aluminum entrance window. 
In order to improve light collection efficiency, direct light collection method was applied by changing the installed position from the center of the case to the inner surface of the entrance window and by removing both acrylic light guides and reflection material coated on the scintillator surfaces.

(3) Performance Test

The performance of the new monitor was tested by using three kinds of quasi-monoenergetic neutron fields with 45, 60 and $75 \mathrm{MeV}$ peaks at TIARA. In the tests, the monitor was set on the surface of the collimator exit (Fig. 3). Sensitivity of the new monitor and linearity of the new monitor's count rate to the beam current were investigated. In order to investigate the correlation of the count rate with neutron beam intensity after passing through the collimator and the new monitor, other detectors (another fission chamber and an organic liquid scintillator) in the irradiation field were used as a reference of beam intensity. The fission chamber was set at $68 \mathrm{~cm}$ from the collimator exit for high beam current. The organic liquid scintillator was set at $9.4 \mathrm{~m}$ from the collimator exit for low beam current. The correlation of the count rate of the new monitor and those of the detectors were investigated. Data on the sum of pulse heights of the two PMTs were taken together with data on the existing monitor and beam current.

(4) Results of Performance Test

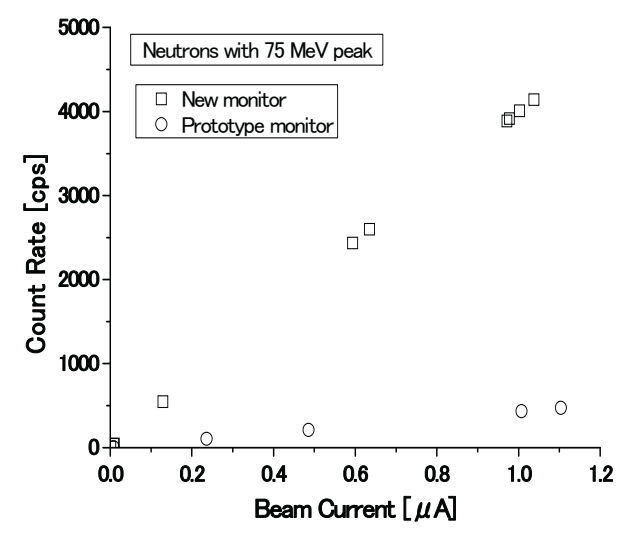

Fig. 4 Count rate v.s. beam current for neutrons with $75 \mathrm{MeV}$ peak. Good linearity of the new monitor's count rate to beam current was seen.

Sensitivity of the new monitor was increased about ten times from that of the prototype by improvement of light collection efficiency, which was the same level as that of the existing monitor (Fig. 4). It was confirmed from the figure that the new monitor's count rate had good linearity to beam intensity (beam current) within the range of interest in ordinary use.

As shown in Fig. 5, the correlation of the count rate of the new monitor with the neutron beam intensity in the irradiation room became somewhat better than those of the existing monitor set ahead of the collimators. As noted in Fig. 5, standard deviations were 1.03 and $2.03 \%$ to each mean value for the new monitor and the existing monitor, respectively.

\section{Summary}

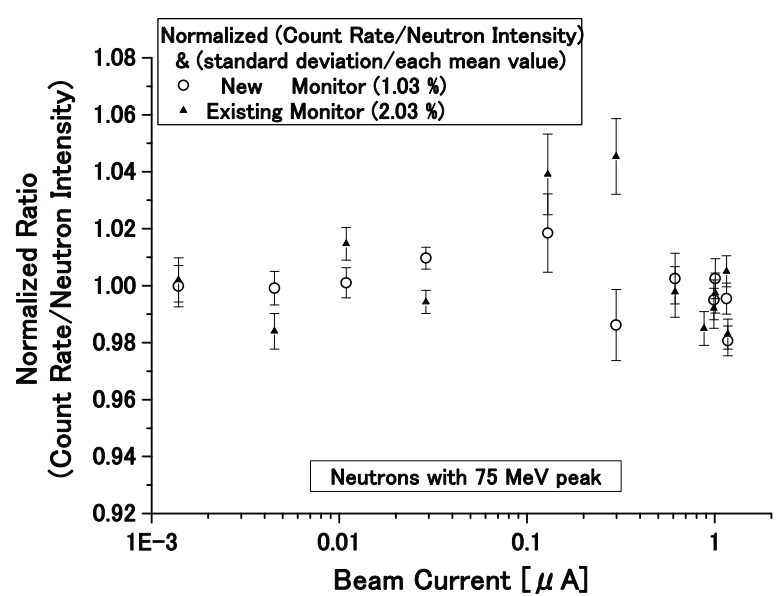

Fig. 5 Normalized ratio of count rate per neutron beam intensity v.s. beam current for neutrons with $75 \mathrm{MeV}$ peak. The correlation of the new monitor's count rate to the neutron beam intensity in the irradiation room became better than that of the existing monitor.

Quasi-monoenergetic neutron calibration fields for several tens of $\mathrm{MeV}$ at TIARA are being developed now. Three neutron fields with 45,60 and $75 \mathrm{MeV}$ peaks are planned to be established, considering the construction of high energy neutron standards and the international intercomparison. As one of the most important characteristics, neutron fluences were measured by using the newly developed PRT with high efficiency. The uncertainties of the fluence were estimated less than $6.5 \%$. For establishment of the fluence monitoring technique, the transmission type neutron fluence monitor with the thin plastic scintillator was developed. As a result of the performance tests, good linearity of count rate to various beam intensities within a range of interest in ordinary use, sufficient sensitivity as the fluence monitor and good correlation with the actual beam intensity in the irradiation room were confirmed.

\section{Acknowledgment}

The authors wish to thank the cyclotron operating staff and Mr. Kaneko of the JAEA-Takasaki for their collaboration.

\section{References}

1) M. Baba, Y. Nauchi, T. Iwasaki, et al., "Characterization of a 40-90 MeV ${ }^{7} \mathrm{Li}(\mathrm{p}, \mathrm{n})$ neutron source at TIARA using a proton recoil telescope and a TOF method," Nucl. Instrum. Meth., A428, 454 (1999).

2) Y. Shikaze, Y. Tanimura, J. Saegusa, et al., "Investigation of properties of the TIARA neutron beam facility of importance for calibration applications," Radiat. Prot. Dosim., 126(1-4), 163 (2007).

3) Y. Shikaze, Y. Tanimura, J. Saegusa, et al., Development of the Neutron Calibration Fields using Accelerators at FRS and TIARA of JAEA, J. Nucl. Sci. Technol., Suppl. 5, (2008) 209.

4) Y. Shikaze, Y. Tanimura, J. Saegusa, et al., Evaluation of the Performance of a New Fluence Monitor for Quasi-monoenergetic Neutron Calibration Fields of Several Tens of MeV Range, JAEA-Review 2008-055, (2008) 166. 